Rhode Island College

Digital Commons @ RIC

\title{
Increasing the Knowledge of Critical Care Nursing Staff Related to Sepsis: A Program Development Project
}

Matthew Willard

Follow this and additional works at: https://digitalcommons.ric.edu/etd

Part of the Critical Care Nursing Commons

\section{Recommended Citation}

Willard, Matthew, "Increasing the Knowledge of Critical Care Nursing Staff Related to Sepsis: A Program Development Project" (2018). Master's Theses, Dissertations, Graduate Research and Major Papers Overview. 273.

https://digitalcommons.ric.edu/etd/273

This Major Paper is brought to you for free and open access by the Master's Theses, Dissertations, Graduate Research and Major Papers at Digital Commons @ RIC. It has been accepted for inclusion in Master's Theses, Dissertations, Graduate Research and Major Papers Overview by an authorized administrator of Digital Commons @ RIC. For more information, please contact digitalcommons@ric.edu. 


\title{
INCREASING THE KNOWLEDGE OF CRITICAL CARE NURSING STAFF RELATED TO SEPSIS: A PROGRAM DEVELOPMENT PROJECT
}

\author{
by \\ Matthew J. Willard \\ A Major Paper Submitted in Partial Fulfillment \\ of the Requirements for the Degree of \\ Master of Science in Nursing \\ in \\ The School of Nursing \\ Rhode Island College
}

2017 


\begin{abstract}
Sepsis can lead the body into organ failure, tissue damage, amputations, and in severe cases, even death. Yale New Haven Health-Westerly Hospital has several new RN's that are at a novice level of knowledge about how to treat and care for the septic patient. Knowledge of the signs and symptoms of sepsis can lead to a quicker diagnosis and improve patient outcomes. The purpose of this program development project was to improve the knowledge of sepsis identification and treatment by providing education to the critical care nursing staff at Yale Westerly Hospital.

A non-probability convenience sampling plan was utilized. Fifteen nurses out of a potential twenty nurses completed the pre-test and post-test component. Each participant's test score from the pre-test in comparison to the post-test improved after the education component of the project was implemented. Pre-test scores encompassing the entire exam, ranged from $35 \%$ to $60 \%$ and post-testing scores ranged from $65 \%$ to $85 \%$. The mean score of the pre-test was $52 \%$ and the mean score of the post-test was $78.6 \%$. The mean scores from the pre-test compared to the post-test improved by $26.6 \%$. More experience in the critical care setting did influence the average test scores in both the preand post-test. APRN's are in a unique position to enable educational programs and assess educational needs of a certain department. The APRN can deliver education not only for the novice nurses, but for all nurses throughout the experience spectrum.
\end{abstract}




\section{Table of Contents}

Background/Statement of the Problem ................................................................. 1

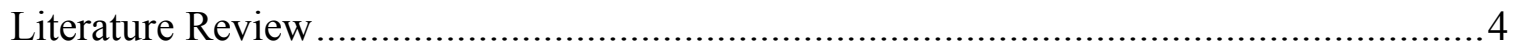

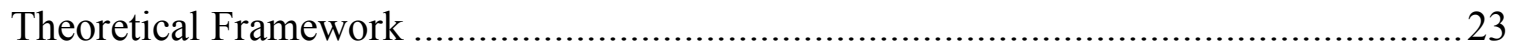

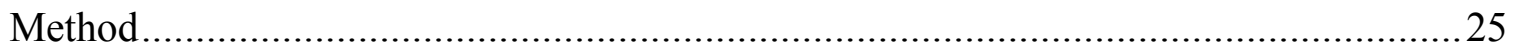

Results.................................................................. 31

Summary and Conclusions............................................... 35

Recommendations for the Advanced Practice Registered Nurse......................37

References................................................................ 38

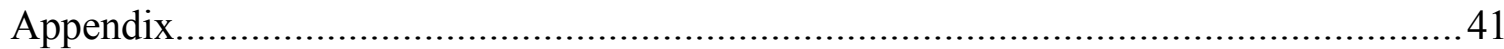




\section{INCREASING THE KNOWLEDGE OF CRITICAL CARE NURSING STAFF RELATED TO SEPSIS: A PROGRAM DEVELOPMENT PROJECT \\ Background/Statement of the Problem}

Yale New Haven Health-Westerly Hospital is a small community hospital in Westerly, Rhode Island. The hospital contains one nine-bed intensive care unit. Most patients admitted to the unit are admitted with a diagnosis of severe sepsis or septic shock. Over the past year the unit hired several new registered nurses that are brand new to the critical care setting and are at a novice level of knowledge about how to treat and care for the septic patient.

A thorough nursing assessment includes identifying the onset of sepsis through specific patient symptoms. Symptoms in the adult patient with diagnosed sepsis includes hyperthermia, tachycardia, tachypnea, decreased level of consciousness or change in mental status, hypotension causing decreased perfusion to the kidneys and low urine output (Peel, 2008). Identifying sepsis in the elderly can be more difficult since the elderly don't always present with the same symptoms as a younger adult patient would (Peel, 2008). For example, the elderly patient may only present with the symptoms of decreased level of consciousness and hypothermia, compared to hyperthermia which the younger adult patient might present with. This can delay diagnosis of the septic patient in

a timely manner which can cause the patient to have a worse outcome. If the nursing staff has expertise about these symptoms they could alert the practitioner and a quicker diagnosis can be made. 
Sepsis treatment protocols are outlines of care used to treat the septic patient. The protocols outline the interventions arranged by time of diagnosis and progresses in conjunction with the severity of symptoms. Starting these protocols in a timely manner can significantly affect these patient's outcomes. Mortality rates from sepsis can increase by up to $8 \%$ every hour that treatment is delayed (Sepsis.org, 2017). Protocols include ordering specific laboratory data including lactate levels, complete blood cell counts, blood sugars, coagulation panels, and blood cultures. Laboratory results can be the main biomarkers when treating these patients. Baseline laboratory results can be used to tailor treatment specific for these patients. The nursing staff need to be aware of what these results signify and how to compare current results to the baseline results and conclude if current treatment is effective and productive especially in antimicrobial therapy.

Hemodynamic monitoring and vasopressor therapy are also included in the protocol if the patient progresses to septic shock and becomes hemodynamically unstable. Hemodynamic monitoring and vasopressor therapy are only done in the intensive care unit so the critical care nursing staff needs to be knowledgeable about hemodynamic monitoring and how to initiate and monitor vasopressor therapy (Peel, 2008).

The septic patient is often being observed and treated around the clock, especially in the first twenty-four hours of diagnosis. The treatment of the patient presenting with septic shock or severe sepsis, should begin in the emergency department and continue in the critical care setting. Critical care nurses need to be aware of sepsis protocols to ensure the protocol is started in the emergency department before transfer of the patient to the critical care setting. Critical care nurses should have a general knowledge of emergency room sepsis treatment so better continuity of care can occur. Sepsis can also occur at any 
point during the patient's admission so knowing how treatment begin is essential to critical care nurses.

Since patients admitted to the unit with a diagnosis of sepsis are so complex, an increase in clinical education will benefit new nurses as well as patients' care and outcomes. There are several different areas of sepsis care in which critical care nurses need to be knowledgeable about, and identifying these early are vital to the patient's outcome. The purpose of this program development project was to improve the knowledge of sepsis identification and treatment by providing education to the critical care nursing staff at Yale New Haven Health-Westerly Hospital. 


\section{Literature Review}

This literature review was conducted using the databases from the years 20062017. The databases included Medline, Pub Med, Google Scholar, and Cumulative Index and Allied Health Literature (CINAHL). Keywords included in these searches were as follows; sepsis protocols, sepsis treatment, sepsis identification, sepsis laboratory data, sepsis and hemodynamics, sepsis and fluid therapy, and sepsis in the elderly.

\section{Sepsis: An Overview}

Background. Sepsis is the body's response to infection. This type of response can lead the body into organ failure, tissue damage, amputations, and in severe cases, even death. The systemic response is manifested by two or more of the following; body temperature greater than thirty-eight degrees Celsius or less than thirty-six degrees Celsius, a heart rate greater than 90 beats per minute, a respiratory rate greater than 20 breaths per minute or a $\mathrm{PaCO} 2$ of less than thirty-two and finally a white blood cell count greater than twelve or less than four (Sepsis.org, 2017).

Septic shock is sepsis accompanied by hypotension despite adequate fluid resuscitation that causes perfusion abnormalities and include lactic acidosis, oliguria, acute mental status changes and others (Robson, 2008). The progression from sepsis to septic shock begins with the inflammation response to the invading pathogen.

Neutrophils then create cellular change and inflammation and vasodilation occurs. The invading pathogen stimulates the release of cytokines which trigger an exaggerated inflammatory response. Next, fluid leaks from circulation into the interstitial tissue which causes hypotension to occur due to a decrease in intravascular volume which leads to 
lactic acidosis and cellular hypoxia. Systemic hypotension is eventually caused by tumor necrosis factors and increased levels of nitric oxide (Dunkley \& McLeod, 2015).

According to Sepsis Alliance 2017, sepsis is currently the leading cause of death in the United States in the acute care hospital setting. More than twenty-six million people worldwide every year are diagnosed with sepsis which translates to an individual being effected every twenty seconds. The incidence rates are rising by $8 \%$ every year (Sepsis Alliance, 2017). Sepsis causes more deaths than breast and colon cancer combined and currently kills more patients than lung cancer. At present, there are no major sepsis charities to help fund the development of sepsis care unlike lung, breast, and colon cancer (Robson, 2008). These statistics are compounded by rapidly increasing complications related to sepsis in our current patient population, which can be attributed to the increasing elderly population. With people living longer, more comorbidities are present which cause the patient to be at a greater risk for sepsis (Robson, 2008). The cost of care for sepsis is vast. Costs in the United States are estimated at approximately twenty-four billion every year. Just one patient in the intensive care unit (ICU) can cost up to $\$ 1800$ daily (Robson, 2008).

\section{Sepsis Protocols}

Background. In 2002, an international operation titled: Surviving Sepsis Campaign was initiated. This campaign was introduced due to the growing concern worldwide about the high death rate of sepsis and protocols were started when suspecting a patient is septic. These protocols are all based on time since timing in sepsis care has a major influence on the outcome of the patient. The protocols include "care bundles" that 
are evidence-based interventions and when implemented together properly, produce a better outcome for the patient (Picard, O'Donoghue, Young-Kershaw \& Russell, 2006).

Once a patient has been diagnosed with sepsis by having two or more of the main signs and symptoms of sepsis, the initial resuscitation phase begins. This phase should begin and be completed in the first three "golden hours" of suspecting the patient is septic. This is usually completed in the emergency room setting but can be initiated at any point during the patient's admission that sepsis is suspected. The protocol begins in the first hour of identifying sepsis and usually continues into the first six hours of care. There are four main goals that need to be achieved to improve the patient's outcome. The main goal of the initial resuscitation phase is to attempt to restore adequate organ perfusion. This goal can be achieved by a central venous pressure (CVP) between 8-12 mmHg. The care team needs to be aware of the patient's fluid status and this can be interpreted by CVP readings. Fluid replacement improves cardiac output, delivery of oxygen, tissue perfusion and overall mortality rate in patients with sepsis. (Picard, O'Donoghue, Young-Kershaw \& Russell, 2006).

Septic shock causes cardiac dysfunction and vasodilation which then leads to organ dysfunction and failure due to hypoperfusion. Due to this phenomenon, the second goal of the initial resuscitation is achieving a mean arterial pressure (MAP) greater than or equal to 65 . The MAP reflects the driving pressure to supply all the body's vital organs. The third goal is monitoring urine output. Urine output also needs to be assessed to assure that adequate renal perfusion and cardiac output is maintained. Urine output greater than or equal to $0.5 \mathrm{ml} / \mathrm{kg} / \mathrm{hr}$ is the standard goal. The practitioner should also 
consider urinary catheter insertion so the nursing staff can calculate a more accurate urine output.

The final goal of the initial resuscitation phase is for the patient to achieve a central venous oxygen greater than or equal to $70 \%$. There are a couple of ways this can be achieved. Once the patient has achieved a CVP reading of greater than or equal to $8 \mathrm{mmHg}$ and if the patient is both hypovolemic and has a hematocrit level less than thirty percent, the practitioner would next order a blood transfusion. If the patient's CVP is less than 8 , then further fluid challenges need to be ordered, prior to ordering transfusions. Blood products increase central venous oxygenation $(\mathrm{ScvO} 2)$ due to increased oxygen delivery to the tissue beds and keeping the CVP pressure greater than $8 \mathrm{mmHg}$ for a longer period than just intravenous fluid alone. Once these four goals have been achieved the next part of the protocol begins which includes laboratory work, antimicrobial therapy, steroid therapy and glucose control, further hemodynamic monitoring, vasopressor therapy and mechanical ventilation if needed (Peel, 2008).

Empirical evidence. Alsawalha et al, (2008) studied the effect of a "sepsis bundle" on 28-day mortality for the treatment of septic shock in older adults. The authors completed an observational prospective study with a historical control group in a tertiary care center. Participants of the study included 87 patients recruited between the years 2004 and 2007, who were treated with the "sepsis bundle" protocol. The authors then matched this group to a historic group of patients treated for sepsis between the years of 2001 and 2004 who were not treated with the protocol. The researchers concluded that the patients who were treated according to the "sepsis bundle" had an absolute risk reduction in 28-day mortality of $16 \%$ compared to those patients who weren't. The group 
of patients that were treated with a protocol received a larger volume of fluid in the first six hours in the emergency department and received lower doses of vasopressors. The authors concluded that according to the cox regression analysis, the implementation of the "sepsis bundle" protocol was associated with a better 28-day survival (El Solh, et al, 2008).

Niazi, et al, (2011) created a study that also measured outcomes following implementation of a standardized protocol for treating patients with severe sepsis and septic shock. An interdisciplinary team was created to educate and improve early recognition of sepsis and severe sepsis/septic shock treatment over a six-month period. Following an education session, the "sepsis bundle" protocols were implemented. The sample included adult patients admitted to the emergency department and/or critical care unit with severe sepsis/septic shock at a 563-bed tertiary care facility between the years 2008-2010. Appropriate recognition of patients with sepsis, and achievement of treatment goals within six hours of onset were measured. The authors concluded that when standardized protocols were utilized, there was significant success associated with meeting "6-hour goals," which include stabilizing the patient hemodynamically (Niazi, et al., 2011).

\section{Sepsis Laboratory Data}

Background. Following oxygen therapy and stabilizing a patient hemodynamically, blood work should be collected. Laboratory data in sepsis is a large component of the therapy. The tests act as biomarkers, signifying if the patient's status is improving or declining. Antimicrobial therapy can also be tailored to the results found and a more appropriate antibiotic than a broad-spectrum antibiotic may be used. 
As a result of tissue hypoxia in sepsis, the patient's serum lactate is usually elevated greater than four mmol/litre. Measuring the lactate level can assess the severity of the tissue hypoxia as well as guide the practitioner's orders for fluid resuscitation. The measurement should be obtained immediately, particularly prior to broad spectrum antibiotics being administered. This measurement will act as a baseline to evaluate the patient's response to antibiotic therapy especially during the first twenty-four hours of care. This will occur by a second level being drawn once the first dose of antibiotic(s) are administered. The level should be evaluated to ensure the level is trending downwards compared to the first level, signifying lactate clearance and an improvement in the patient's condition. Early lactate clearance in the course of therapy is associated with decreased mortality (Vaughan, \& Parry, 2016).

The other primary laboratory test that should be ordered are two sets of blood cultures. The test should also be ordered prior to the patient's first dose of antibiotics. The cultures can help identify the specific kind of bacteria present and provide information for the practitioner to choose the most appropriate antibiotic. Furthermore, all infected sources should be removed with positive results including removing any catheters, cannulas or other infected equipment from the patient. If the infected source is necrotic tissue or an abscess, surgical intervention must then be ordered (Hansen, 2013). Cultures from other suspected sources should be considered as well including urine samples, sputum samples, wound samples, and cerebrospinal fluid sample.

Serum cortisol levels are drawn on admission and if the patient does not have an increase in cortisol greater than $248 \mathrm{nmol} / \mathrm{L}$, steroid replacement should be ordered for seven days. Glucose levels should also be monitored closely since these levels tend to 
increase with infection and can further lead to organ damage, especially in the diabetic patient. Around the clock monitoring and insulin therapy including insulin drips may be necessary. Tight glucose control has been linked to a decrease in the mortality rate for the septic patient by preserving organ function. Other lab tests that should be considered are complete blood cell count with differential, chemistry panel every six hours, liver function tests, $\mathrm{C}$ reactive protein, procalcitonin level, international normalized ratio, prothrombin time, and partial prothrombin time (Hansen, 2013).

Empirical evidence. Procalcitonin levels are considered in the treatment of sepsis. Jensen, et al, (2006) investigated whether daily changes in procalcitonin and maximum obtained levels could act as predictors of mortality in critically ill patients. A prospective observational cohort study was completed which included four hundred seventy-two patients with diverse comorbidities and age who were admitted to a critical care unit at a tertiary care hospital in Denmark. The interventions ordered for the treatment were equal in all patient groups, and antimicrobial therapy was adjusted according to the procalcitonin levels. Daily procalcitonin levels as well as white blood cell counts and C-reactive proteins were drawn on 472 critically ill patients. The authors concluded that a high maximum procalcitonin level and an increased procalcitonin for one day were independent predictors of an all-cause-mortality in a 90-day period using the multivariate cox regression analysis model. An increase in C-reactive protein or leukocytes did not show these results (Jensen, et al, 2006).

Marty et al (2013), conducted a prospective observational study in a surgical ICU to investigate the prognostic value for death based on lactate clearance. The study collected data during the first 24 hours in of sepsis treatment. The authors measured 
blood lactate concentrations upon admission to the hospital and at 6 hours, 12 hours, and 24 hours later. The researchers found that the mean time between the diagnosis of severe sepsis and admission to the ICU was approximately 4.5-8.0 hours. Logistic regression concluded that the first 24 hours lactate clearance was independently correlated to survival status and was the best parameter associated with mortality rate in septic patients. Lactate clearance-directed therapy should be considered in septic patients, even after the first 6 hours of treatment (Marty, et al, 2013).

Early action against sepsis is a standard of care in many acute care settings and the role of different health care providers is well recognized. However, the impact of a telephone call from a specialist in the microbiology clinical setting when a patient has positive blood cultures has not been assessed. Bouza, et al, (2015) investigated if telephone calls from a specialist in the laboratory to providers regarding blood culture status improved patient care. The phone call was then followed by an interview with health care providers caring for adult patients whose blood cultures came back positive for bacteria (Bouza, et al, 2015). The study included 300 patients who were suspected of sepsis as well as the nurses and physicians who oversaw the patient's care. Patients were randomly placed in two different groups. Group A had a telephone call performed and group B no telephone call occurred. At the end of the intervention for group A, recommendations on the use of microbiology tests as well the management of the results including antibiotic choice were discussed. The median number of days of antibiotic use in groups A and B were, respectively, 6 days versus 9 days and the median number of prescribed daily doses of antimicrobials were lower in group A. The authors concluded that an immediate telephone call after the findings of positive blood cultures, with 
suggestions for interventions from the microbiology laboratory to the health care

provider, improved the recognition of sepsis and the use of diagnostic resources. Making a telephone call and discussing antibiotic therapy also reduced antimicrobial use which decreases expenses (Bouza, et al., 2015).

\section{Hemodynamic Support}

Background. Maintaining the stability of the septic patient's hemodynamic status is one of the main goals in sepsis care. When the patient is first suspected to have sepsis, intravenous fluid challenges should be initiated. The patient can receive on average 6-8 liters of crystalloid fluids in the first three hours of care alone. The practitioner must also be aware of adverse effects of therapy such as pulmonary fluid overload especially in the cardiac impaired patient. Hemodynamic monitoring, including right arterial monitoring, pulmonary artery monitoring and arterial lines are also used. The septic patient is an everchanging clinical emergency so having these continuous readings are very beneficial to the practitioner and nursing staff (Perez, 2015). Initial fluid challenges in patients with tissue hypoperfusion due to sepsis should be $30 \mathrm{ml} / \mathrm{kg}$ of crystalloid fluids. Albumin in the resuscitation phase of sepsis care can also be used if the patient is requiring a substantial amount of crystalloid fluid (Rhodes, Evans, Alhazzani, et al., 2016).

Empirical evidence. Borer, et al, (2016) collected data from 297 hospitalized patients with sepsis in a medical ICU between the years 2005 and 2011. The patients were then divided into four groups, group one included patients with fluid balance at discharge (FBD) that was less than 10 liters; group two included patients with an FBD between 10 to 20 liters; group three included patients with an FBD between 20 to 30 liters; and group four included patients with FBD greater than 30 liters. The mortality rate 
was significantly higher in groups two through four compared to group one. The authors suggest that positive cumulative fluid balance is a major factor that can predict the clinical outcome of critically ill septic patients in the critical care setting as well as after discharge (Borer, et al, 2016).

Fluid administration continues throughout the treatment of the septic patient if the patient's hemodynamic status is improving and patient is tolerating the fluid. Hariyanto, Yahya, Widiastuti, Wibowo, \& Tampubolon, (2017) explored both the benefits and disadvantages that fluid challenges cause during sepsis care in a case report on an 86year-old man with no prior comorbidities. The researchers found that an average of four liters of fluid was administered during the first six hours of diagnosis and within the first 72 hours, more than eight liters were administered. The patient discussed in the study received four liters of crystalloid infusions, nevertheless the patient's MAP remained below 65 and vasopressor therapy was initiated. The patient's urine output was less than $1 \mathrm{ml} / \mathrm{kg} / \mathrm{hr}$ and the patient's creatine levels were rising daily (Hariyanto, Yahya, Widiastuti, Wibowo, \& Tampubolon, 2017).

The authors point out that the nursing staff and practitioners need to be cautious of continuing intravenous fluids. Further fluid therapy could increase edema. With progressive capillary leak, the kidneys and liver are unable to compensate for the increased volume. Increased edema leads to microvascular congestion and decreased peritubular flow which caused the patient in the study's acute renal failure. The authors conclude that starting vasopressors at an earlier time can have a beneficial outcome for the patient. Acquiring an adequate MAP can cause adequate organ perfusion and 
decrease the formation of edema (Hariyanto, Yahya, Widiastuti, Wibowo, \& Tampubolon, 2017).

Current evidence on fluid therapy and sepsis care should urge practitioners to reconsider the regimen of fluid management. Aggressive fluid management after the resuscitation phase in sepsis care has well-documented poor outcomes for the patient. Some patients respond poorly to these fluid challenges because arteriovenodiliation and microcirculatory dysfunction occurs, therefore fluids during the resuscitation phase are best given with early vasopressor therapy. After the patient is stabilized in the initial resuscitation phase, these fluids should be tapered to prevent fluid overload which will worsen oxygen transport. The authors further discuss how complex sepsis treatment is and how practitioners need to be more aware of the possible poor outcomes that intravenous fluid therapy can have on the patient (Hariyanto, Yahya, Widiastuti, Wibowo, \& Tampubolon, 2017).

The benefit of fluid resuscitation was also questioned in an observational cohort study by Sakr, et al, (2017). The study included 730 intensive care units in 84 countries. Patients were all adult patients admitted to the ICU between May 8 and May 18, 2012. This excluded admissions for routine postoperative surveillance. In this retrospective study the authors included 1,808 patients that were admitted to the ICU with an admission diagnosis of sepsis. Patients were then stratified per quartiles of cumulative fluid balance between 24 hours and 3 days after their admission to the unit. The study found that ICU and hospital mortality rates were $27.6 \%$ in the first 24 hours and reached $37.3 \%$ within the first three days of admission. In the first three days, cumulative fluid balance increased from $1,217 \mathrm{~mL}$ in the first 24 hours to $1,794 \mathrm{~mL}$ on 
day three. There was little difference found in the cumulative fluid intake between survivors and nonsurvivors, but fluid balance was less positive in survivors. In survivors, fluid balances were in the negative after the third ICU day but were positive in nonsurvivors. The study included that there was a stepwise increase in the death rates the longer the patients needed fluid resuscitation. So fluids should be discontinued as soon as the patient is hemodynamically stable without them. The authors mentioned that early vasopressor therapy initiation can help prevent the adverse effects of fluid therapy and help improve mortality rates (Sakr, et al, 2017).

\section{Adjunctive Pharmaceutical Therapy}

Background. When intravenous fluids are not enough for the patient to become hemodynamically stable, vasopressor therapy should be started and titrated to a MAP of $65 \mathrm{mmHg}$. There are different types of vasopressors for treating septic shock. One medication that is found to have the best outcome for the septic patient with the least amount of side effect is norepinephrine. Norepinephrine should be the practitioner's first choice. Epinephrine should be the second choice when a second vasopressor is needed and can sometimes be substituted for norepinephrine. Dopamine is an alternative vasopressor agent that is rarely used however due to the increased risk of tachyarrhythmias especially in the cardiac compromised patient (Rhodes, Evans, Alhazzani, et al., 2016).

Empirical evidence. Dopamine and norepinephrine were tested for effectiveness in a multicenter, randomized trial (De Backer et al., 2010). The authors assigned 1679 patients with septic shock to receive either dopamine or norepinephrine as first-line vasopressor therapy to restore and maintain blood pressure. Secondary vasopressors were 
added if the singular medication was not enough. The rate of death after 28 days after randomization was the primary outcome measured. The results concluded that there was no significant difference in rate of death at 28 days. Although there was no significant difference in the rate of death between patients, those patients who were treated with dopamine had many more adverse effects including arrhythmias (De Backer et al., 2010).

The guidelines for the adrenergic support of septic shock are considered controversial. Making it difficult for the practitioner to choose the appropriate vasopressor for the septic patient. The practitioner needs to take into consideration that every patient is different so what is appropriate treatment for one patient might not be for the next patient. Norepinephrine is still considered the optimal treatment option for septic shock when vasopressors are instituted. However, a study which investigated the impact of vasopressor support choice on the mortality of the patients with community-acquired septic shock concluded otherwise (Carneiro, Pereira, Póvoa, \& Ribeiro, 2009). The study was a cohort, observational study in seventeen Portuguese critical care units. Patients were all adults and were admitted for septic shock between the years of 2004 and 2005 . Patients were followed during the first five days in the critical care unit, the day the patient was discharged or death resulting, and hospital outcome. The 458 patients in the study received norepinephrine (73\%) and dopamine (27\%). The group of patients who received norepinephrine were found to have a higher hospital mortality. A Kaplan-Meier survival curve showed reduced 28-day survival in the norepinephrine group. The authors concluded that patients with community-acquired septic shock who received norepinephrine administration could be associated with worse outcome (Carneiro, Pereira, Póvoa, \& Ribeiro, 2009). 
A similar study done by Agrawal, Consul, Gupta and Shastri (2011), which compared the capability of norepinephrine and dopamine in reversing hemodynamic and metabolic instability in the septic shock patient concluded different results. The authors completed a prospective randomized control study consisting of fifty patients presenting with septic shock that needed vasopressor therapy. The goal of therapy was to achieve as well as maintain hemodynamic stability within six hours. Two groups were created randomly and one group was started on dopamine and the other on norepinephrine. The authors found that the group who received dopamine showed an increased heart rate and a significant higher cardiac index of oxygen delivery compare to the group who received norepinephrine. The group that received norepinephrine had a higher mean systolic blood pressure as well as a higher systemic vascular resistance index. The authors found that norepinephrine was more beneficial in reversing hemodynamic and metabolic abnormalities in the septic shock patient (Agrawal, Consul, Gupta, Shastri, 2011).

\section{Sepsis in the Elderly}

Background. Like most diseases, sepsis effects individuals differently. One main patient population that may present differently and differ in treatment management are the elderly population. The practitioner needs to be aware of this special population and how sepsis identification and treatment are different in comparison to treating the younger septic patient.

It is predicted by the year 2030 that one out of every five Americans will be aged 65 years or old with the greatest growth in the 85 years and older population. Therefore, emergency departments and acute care settings can expect to see an influx of geriatric patients. Among these geriatric admissions, sepsis accounts for $20 \%$ of all geriatric 
admissions currently (Aminzadeh, \& Parsa, 2011). Practitioners and nurses might have a difficult time identifying sepsis in this population because sepsis in the elderly usually goes unnoticed until the patient is in severe sepsis and septic shock. The elderly usually exhibit certain signs and symptoms that the practitioner and nursing staff should notice and make a diagnosis as quick as possible. These signs and symptoms include weight loss, failure to thrive, falls, and unexplained changes in mental status or functional ability. The elderly do not always present with high fevers and may be hypothermic or only exhibit low-grade fevers. White blood cell counts may be decreased, or only show a slight raise. Collecting a proper health history is important with geriatric patients, especially if they have had any recent infections or recent hospitalizations that could explain a bacterial infection returning (Aminzadeh, \& Parsa, 2011). The influence on age is overall not understood well and practitioners need to be proactive in diagnosing these patients if sepsis is suspected. Age alone could be a factor in diagnosing sepsis.

Empirical evidence: Elderly patients account for $12 \%$ of the United States population currently and $64.9 \%$ of sepsis cases, yielding a relative risk of 13.1 compared to younger adult patients. Elderly patients are also more likely to have Gram-negative infections associated with pneumonia particularly and have other comorbid medical conditions that compound the infectious process. Martin, et al (2013), sought to determine if age alone had any effect on the incidence, severity, and outcome of sepsis. The authors completed a longitudinal observational study using national hospital discharge data of over 500 acute care hospitals. The study consisted of over ten million adult patients with a diagnosis of sepsis who were hospitalized over a 24-year period. Case-fatality rates were found to increase linearly by age. Age was also found to be an 
independent predictor of mortality in an adjusted multivariable regression. Septic elderly patients died earlier during hospitalization, and survivors were more likely to be discharged to a subacute facility than a younger adult would. The authors concluded that the incidence of sepsis is increased in elderly adults, and age is an independent predictor of mortality. Compared with younger septic patients, elderly nonsurvivors of sepsis died earlier during hospitalization and frequently required skilled nursing or rehabilitative care after hospitalization prior to expiring. The findings could be used for patient care and resource prioritization in the health care system (Martin, et al, 2006).

In the elderly, differentiating sepsis from other causes of systemic inflammation is often problematic. A study was completed that evaluated the ability of C-reactive protein (CRP), procalcitonin (PCT), Erythrocyte Sedimentation Rate (ESR), and Interleukin-6 (IL-6) to help identify sepsis in the elderly patient. The study was a single center prospective observational study which included all consecutive elderly patients who were admitted with alleged sepsis and systemic inflammatory response syndrome (SIRS). Blood samples for measuring CRP, PCT, IL-6, ESR and white blood cells (WBC) count were collected on day one of admission. Specificity, sensitivity, negative and positive predictive values were considered for each value. The authors concluded that CRP exhibited the greatest sensitivity and negative predictive value. CRP also performed best in distinguishing patients with sepsis from SIRS. It was also found that IL-6 performed the best in distinguishing between SIRS and the control group. However, both CRP and ESR were the most accurate diagnostic value for distinguishing sepsis from SIRS in the elderly patient (Talebi-Taher, Babazadeh, Barati, \& Latifnia, 2014). 
Cognitive impairment in the elderly is one of the major determinants of caregiving needs post discharge and can put a strain on the societal healthcare costs. The number of patients with cognitive impairment after being discharged for the treatment of sepsis is high. A prospective cohort study was done to determine the cognitive impairment and physical changes in functioning that can occur after a patient survives sepsis. The study included 1520 older adult patients who were hospitalized for severe sepsis between the years of 1998-2006. The patients studied had a mean age of 76.9 years of age. A comparison group was created that included 5574 patients who were hospitalized for non-sepsis conditions. Interviews were conducted with the patients to assess cognitive impairment using validated modified telephone interview for cognitive status and informant questionnaire on cognitive decline in the elderly. Disability was calculated by the number of activities of daily living and instrumental activities of daily living that the patients needed assistance with. The authors found that the prevalence of moderate/severe cognitive impairment was found to be increased by $10.6 \%$ amongst patients who survived severe sepsis. Also, an increased rate of new functional limitations was found with those patients who had no limits before sepsis. In contrast, those patients hospitalized for non-sepsis conditions were associated with minimal change or no change in cognitive impairment. The authors concluded that severe sepsis in the elderly population was associated with substantial new cognitive impairment and functional disability (Ely, Iwashyna, Langa, Smith, 2010).

\section{Nursing Education on Sepsis}

Empirical evidence. Nurses can also have a significant role in identifying patients with sepsis. Their constant interactions with the patient can prove valuable when 
identifying symptoms early when the patient is becoming septic. Integrating sepsis screenings and protocols as part of the nurse's routine assessment can help identify at risk patients and begin interventions earlier. A study conducted in New Zealand was used to raise awareness amongst staff and improve the management of sepsis by integrating sepsis screenings into the facilities nursing assessment (Kleinpell, 2017). Educational seminars were implemented for the nurses. Education topics of intravenous fluids, lactate, oxygen, blood cultures, antibiotics and urine output were implemented. The authors of the study concluded an improvement in the number of sepsis protocols that were started on patients, increasing from $29 \%$ to $69 \%$. The study proved that having a nursing staff that is more educated on sepsis identification and treatment can result in better sepsis identification and treatment (Kleinpell, 2017).

Nurses in the intensive care unit (ICU) setting play a large role in the detection, prevention and therapeutic interventions for the patient with sepsis. A study by Yousefi, Nahidian, \& Sabouhi (2012) reviewed the effects of an educational program that considered the knowledge, attitude, and practices of ICU nurses. The authors used a quasi-experimental study which consisted of 64 nurses that had at least one year of ICU experience. Scores of attitude, knowledge and practice of the participants were reviewed through a questionnaire before and after a one-day educational workshop on sepsis. The findings were compared to the baseline and there was a significant increase in the mean scores of knowledge, attitude, and practice in the group. The authors concluded that the educational work shop significantly improved the levels of knowledge, attitude, and practice of the ICU nurses in sepsis care. Therefore, continuous education on sepsis care should be recommended for healthcare providers (Yousefi, Nahidian, \& Sabouhi, 2012). 
Another study was conducted by Delaney, Friedman, Dolansky \& Fitzpatrick (2015) on nursing competence to determine the influence of a sepsis education program on nurses perceived ability to accurately identify, intervene and care for a patient with sepsis. The program was a multimodal design which included online interactive educational presentations on sepsis including videos and interactive case studies. Pre- and post- testing was completed on the nurse's knowledge as well as medical simulation scenarios. The sample included eighty-two critical care and emergency room nurses with at least one year of experiences working as a nurse in these two fields. The authors concluded that the participants felt more competent in their sepsis care and post-test knowledge scores showed significant improvement (Delaney, Friedman, Dolansky, \& Fitzpatrick, 2015). 


\section{Theoretical Framework}

For the implementation of this program development project a theoretical model that proved to be beneficial was the Donabedian Model. This model provides a framework for exploring health care services and evaluating the quality of care. There are three main components of the model. These components include structure, process and outcomes. The structure component includes all the factors that affect the setting in which care is being delivered which can include the facility, human resources, equipment, as well as staff training. These factors control how patient and providers in a healthcare system act and are considered measures of the average quality of care within a facility (McDonald, Sundaram, Bravata, et al, 2007). Structure may be the cause of problems identified in the process. The structure of this project will be the Yale New Haven Health-Westerly Hospital's intensive care unit where the patient's care occurs.

The process component is the action that make up healthcare such as treatment, diagnosis, patient education, and preventative care. According to Donabedian, the process measurement is nearly equal to the measurement of the quality of care because the process contains all the acts of the delivery of healthcare. Information about process can be collected from interviews with patients and practitioners, direct observation of a health care visit, or from medical records (McDonald, Sundaram, Bravata, et al, 2007). The process component for this project includes the knowledge of the nursing staff specifically about the care of the septic patient.

The outcome of the model contains the total effects of healthcare on patients and includes changes to their behavior, knowledge, and health status, as well as health-related quality of life and patient satisfaction. The outcome stage is considered the most 
important indicators of quality because improving the patient's health status is the primary goal of healthcare. However, accurately measuring outcomes can be very difficult because drawing connections between process and outcomes requires a large sample populations and long-term follow up since outcomes may take considerable time to become observable. The outcome of this project considers if the education provided to the staff was effective or not based on post testing results. The staff will learn new information about sepsis that can be translated into their practice of patient care, thus improving patient outcomes. This education in the long-term will hopefully improve the nurse's care of the patient being admitted with septic shock. The model's domains have advantages and disadvantages that require researchers to draw connections between the domains of the model to identify causation when then is useful for understanding the system as well as designing interventions and experiments in the future (McDonald, Sundaram, Bravata, et al, 2007). 


\section{Method}

\section{Purpose}

The purpose of this program development project was to improve the knowledge of sepsis identification and treatment by providing education to the critical care nursing staff at Yale New Haven Health-Westerly Hospital.

\section{Sample/Setting}

This project was completed in the month of January 2018 at Yale New Haven Health-Westerly Hospital in the intensive care unit. The intensive care unit is a nine-bed unit that can be described as a medical intensive care unit. A non-probability convenience sampling plan was utilized. The sample size included fifteen registered nurses who consented to participate. The inclusion criterion was that the participating nurses had to specifically work in the critical care unit. Float nurses and non-nursing staff were excluded from this sample

\section{Procedures}

The project was verbally approved by the unit manager. Permission from the chief nursing officer was confirmed and approval for this project was granted at the facility (Appendix A). Pre- and post-questions lists were constructed using the book Pass $C C R N !$ Questions were then presented to the unit manager for feedback regarding the test questions. The unit manager was consulted, as an expert regarding sepsis, and asked to make suggestions or changes to test questions. The unit manager approved the questions and did not suggest making any changes. Flyers were posted around the unit one week prior to beginning the testing period with information regarding the purpose of the project as well as a timeline of events that included the testing and education 
component (Appendix B). The flyer included multiple ways of contacting the author if questions were to arise prior, during, or after the project had been implemented. The flyer explained that participation was completely optional and consent would be achieved if the nurse completed the pre-test questions for the project. The day the flyers were posted, an email was sent out using the Westerly Hospital email system including all nurses’ emails who work on the critical care unit (Appendix C). The email explained the project's intent as well as the contact information on the flyer just in case the nurses were unable to view the flyer prior to the project's start date.

The program development project consisted of a pre- and post-education test where the nursing staff began by completing a twenty item-multiple-choice test which evaluated their current knowledge on the topic of sepsis identification and treatment (Appendix D). The tests were composed of questions using the Pass CCRN! test bank. These pre-testing questions provided a baseline to go by and helped aid in considering the nursing staffs' strengths and weaknesses to ensure best identification of education material. Learning objectives for this project can be found in Appendix E.

In the break room on the unit there was station set up that included a designated envelope with copies of the pre-test as well as a designated box with a closed lid for the finished pre-tests to be placed in. The box was emptied periodically throughout the week of the distribution of the pre-tests. Tests collected throughout the week were stored in a locked locker in the break room. This information was also included in the email sent prior to testing.

To ensure confidentiality and provide a means for comparing pre- and post-tests, participants used their mother's month and day of birth as identifiers. The nurses did not 
put their name on the pre- or post-test. On both tests, the staff wrote down the amount of years of critical care experience they had. Experience was compared using less than five years, five to ten years, and more than ten years of critical care experience when reviewing the data collected.

Next, an educational program was presented about severe sepsis and septic shock identification and treatment. The packet highlighted notes for the staff to review on the topic of sepsis identification, management, and treatment. The material was all hard copy and put together into individual binders for the nursing staff to review on their own. The binders were placed in the breakroom in the designated station where the pre-tests were. The bulletin boards in the break room also displayed information that the nurses could review.

Since the profession of nursing is such a demanding and hectic profession, the education provided to the staff was completed on their own time. Letting the staff choose when they would like to complete the education gave them some flexibility in their education and they did not have to worry about making time for a scheduled presentation. After a one-week period, a post test was conducted including the same multiple choice questions on the pre-test. Similar to the pre-test administration, the post-test was available to the staff for completion in a one week period. Both tests contained the same questions so the two tests could be compared. After post testing was completed the two tests were analyzed for data, and answers with rationales for the nurses to review were distributed to the nurses in their break room (Appendix F). The information gathered from the pre- and post-tests gave the author data to determine whether learning had occurred. The data was stored on a flash drive that was stored in the locker room in a locked locker. After all the 
necessary data was collected the two tests were then shredded and disposed of. The information on the flash drive was kept in a locked locker until the completion of the project and then was erased. The flash drive was used on a password guarded laptop.

\section{Measurement/Data Analysis}

The mean scores were compared in several different ways to make final conclusions. First, the scores were assessed by how many of the staff's scores improved from the pre- and post-test. This calculation was done by using the date of their mother's birthday to match pre- and post-tests. The scores were also assessed by years of experience. The mean scores on the pre-test and post-test were then compared by those brackets to investigate how experience played a role in sepsis education.

\section{Desired Outcomes}

The desired outcome for this program development was for the staff's knowledge to improve, as noted with an increase in score from the pre-test to the post-test. Topics that can be identified that may need further education can be relayed to the clinical education department at the facility.

\section{Ethical Concerns}

Ethical concerns included making sure the testing results were kept anonymous. Questionnaires were only identified by mother's birthdate. Year born was not included, just month and day, since this could be used to possibly identify the age group of the participant. IRB approval was granted through Rhode Island College. CNO approval (Appendix A) and unit manager approval were also granted. The CNO and unit manager were kept informed on the progress of the project as it transpired. All data collection was stored on an encrypted flash drive and kept in a locked locker in the nurse's break room 
that only the researcher had access to. All data collected was destroyed upon completion of the program development project.

\section{Organizational Factors}

Administration, including unit manager and chief nursing officer approved the project to be completed at the critical care unit at Yale New Haven Health-Westerly Hospital. The head of the education department also stated support and provided space in the educational office for an area to complete data collection and other work on the project if needed. The unit is very busy so staff could have been apprehensive about completing this type of testing during their shift. Therefore, the staff could take the tests and education with them and then return the final test back to the designated area once complete. Completing this type of education on the staff's own time instead of designating a scheduled time gave the staff more flexibility in their learning. Since the staff was not monitored during the testing period, the result may be skewed if outside resources or other assistance is used. However, it was stressed that testing is completely confidential so there was no pressure to do well. It was also stressed that this project is for the staff's benefit in helping them improve their education on a diagnosis that is seen frequently in their practice setting, Since the staff was not compensated for this education, they may have chosen not to participate. Participation was completely voluntary so the staff did have the right to refuse to participate. Emailing the staff prior to administering the pre-testing and education was an attempt to inform the staff and encourage participation. The staff had contact information provided if they had any questions or concerns about the testing or education processes. 


\section{Evaluation}

Project success was determined by the results from both tests being compared to evaluate whether the project was successful in educating staff on sepsis in the critical care setting. The participants expressed approval of the education project and need for this type of education since the nurse's care for the patient with sepsis and septic shock frequently on the unit. Nurses told the author "those questions were very hard, but the education really helped me in my nursing care." The author was satisfied with the statements about the testing being difficult since if the participants were stating the test was “too easy," than they wouldn't be learning anything and they wouldn't be challenged. 


\section{Results}

Fifteen of a possible twenty nurses $(\mathrm{N}=15,75 \%)$ completed the pre-test, the educational part and the post-test component of the project. All twenty questions on the pre- and post-test were multiple choice questions and for the fifteen pre- and post-tests that were completed, all items were answered on both tests by all participants.

Results of the participant's pre- and post-test scores are illustrated below in Table 1. In Table 2 and Table 3, results are broken down by years of experience in the critical care setting. The two different critical care experience brackets that were used were 1-5 years of experience and $>10$ years of critical care experience.

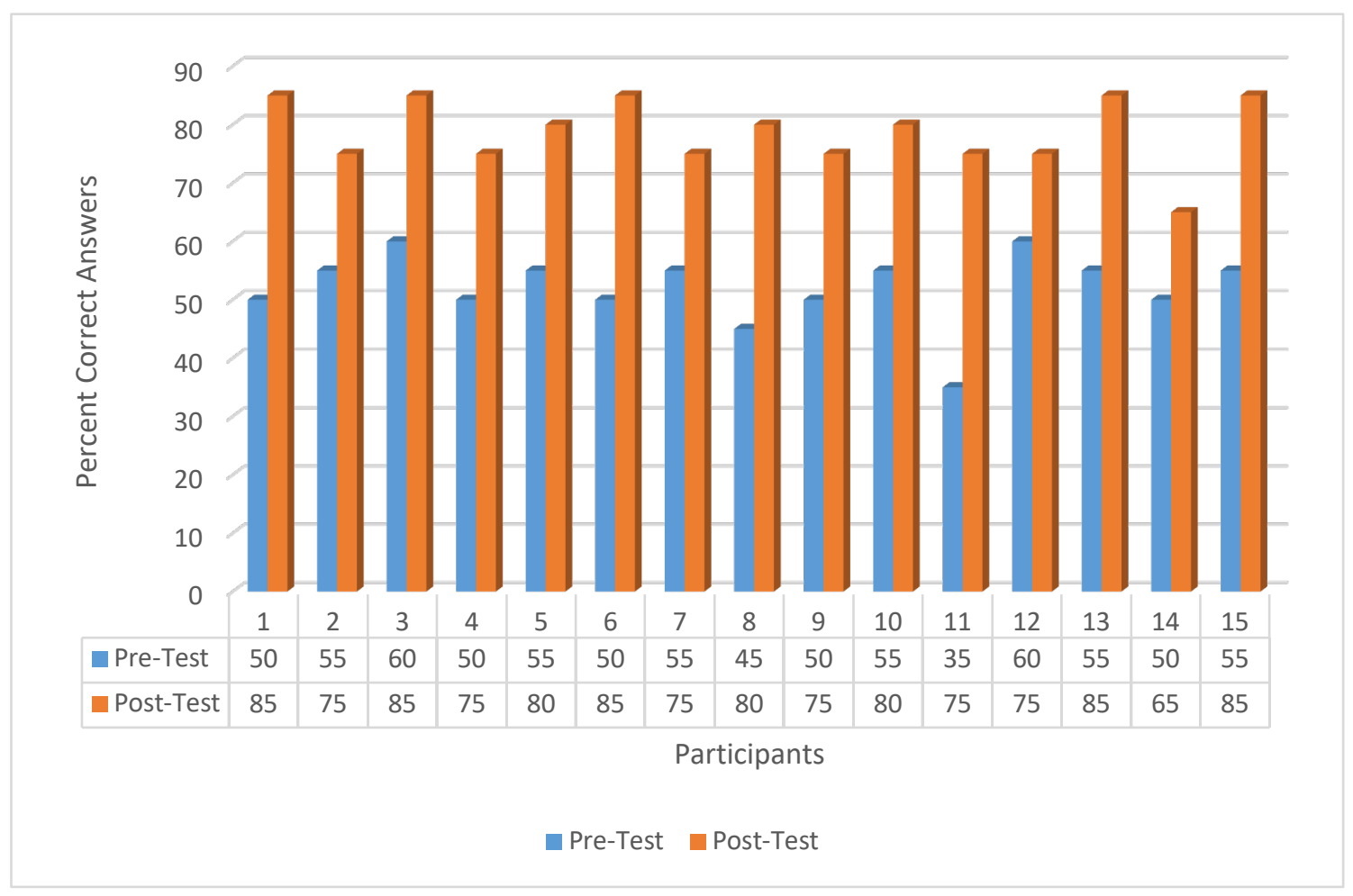

Figure \#1. Test Comparison Pre/Post-Testing

Each participant's test score from the pre-test in comparison to the post-test improved after the education component of the project was implemented. Pre-test scores encompassing the entire exam, ranged from $35 \%$ to $60 \%$ and post-testing scores ranged 
from $65 \%$ to $85 \%$. When evaluating the mean scores of the entire exam, the mean total score of the pre-test was $52 \%$ and the mean total score of the post-test was $78.6 \%$.

Overall, the mean total scores from the pre-test compared to the post-test improved by $26.6 \%$. The biggest improvement was from the pre-test score of $35 \%$ and then a post-test score of $75 \%$. The median total score of the pre-test was $55 \%$ and the median total score of the post-test was $80 \%$, which improved by $25 \%$. The mode total score of the pre-test was $55 \%$ and the mode total score of the post-test was $75 \%$, and improvement by $20 \%$.

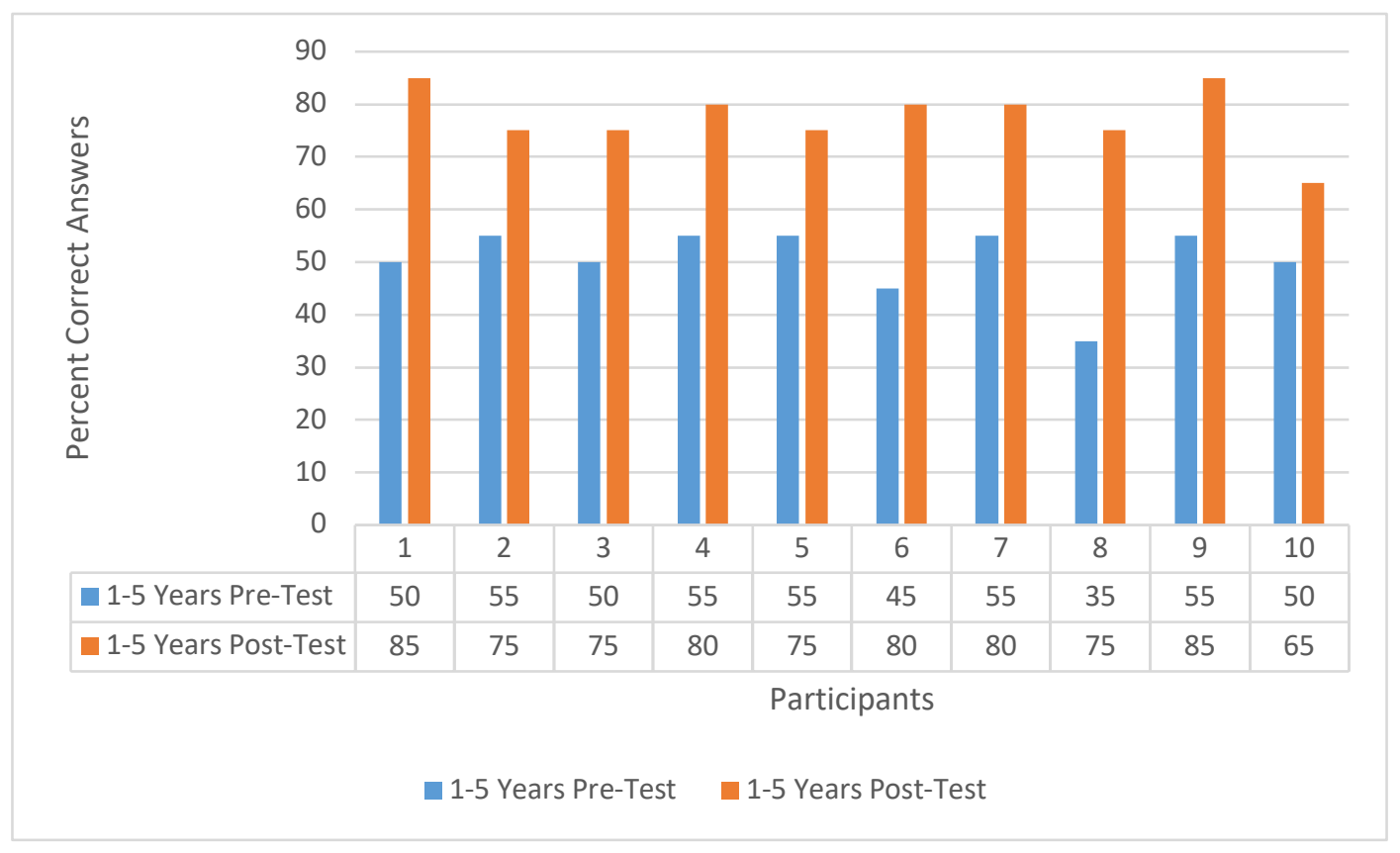

Figure 2. Test Comparison Pre/Post-Testing for 1-5 Years of Critical Care Experience 


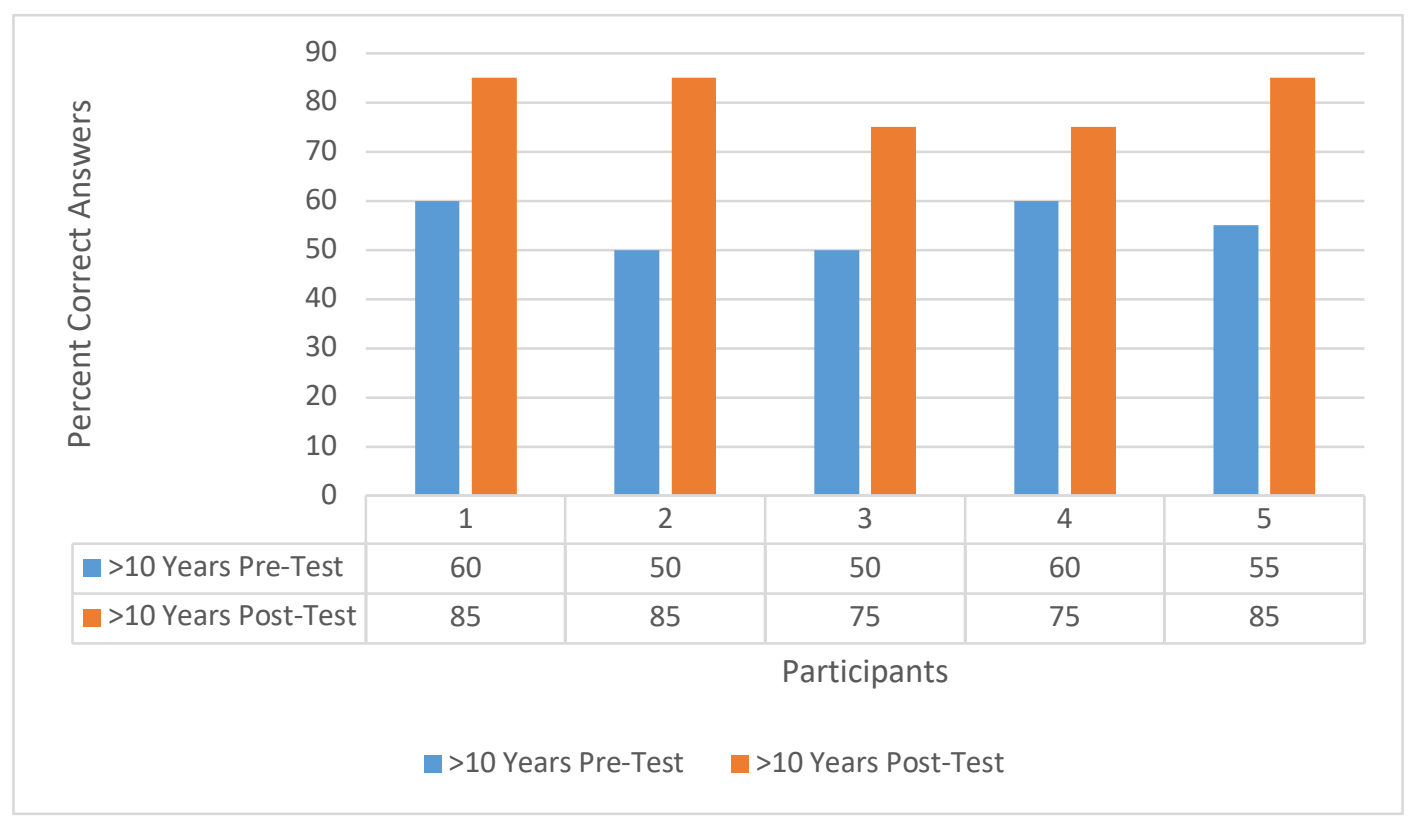

Figure 3. Test Comparison Pre/Post-Testing for $>10$ Years of Critical Care Experience

The scores of the pre- and post-test were also evaluated based on years of experience. The goal of evaluating the scores in this manner was to conclude whether having more critical care experience in sepsis care could have any effect on the scores of the testing. On the pre- and post-test, the participants had to mark in provided brackets how much experience of critical care they had at that time of taking the test. The brackets were broken down into less than one year, 1-5 years, 6-10 years and greater than 10 years. When reviewing the pre- and post-tests it was found that all fifteen participants fell into just two brackets, which were " $1-5$ years" and ">10 years," When comparing the results, it was concluded that the mean score of the ten participants in the " $1-5$ years" of experience for the pre-test was $50.5 \%$ and the post-test mean was $77.5 \%$. Comparing the mean score of the five participants in the ">10 years" of experience for the pre-test was $55 \%$ and the post-test mean score was $81 \%$. When comparing these two mean scores of the two different brackets based on critical care experience it could be concluded that 
having more experience in the critical care setting did influence the average test scores in both the pre- and post-test.

\section{Program Evaluation}

This project has met all the objectives of the project stated in Appendix F. The staff nurses seemed very interested and enthusiastic about having the opportunity to become more educated in the topic of sepsis since many of them were new to the critical care setting. All testing and education was completed on the participant's own time since nursing is a very busy profession. Having the testing being completed on the participant's own time and not in a controlled testing environment could possibly cause participants to use outside resources or each other. A recommendation could be made if this project was to be completed again in the future it may be beneficial to be done under a controlled testing environment. However, the comments from the staff nurse about the project to the author were that they enjoyed being able to complete the testing and education on their own in the comfort of their own home and not have to stay late at work to complete this task. If the testing was done in a controlled testing environment, this could have affected the amount of participation by the staff members. Since all the test questions were taken from a practice CCRN book, hopefully this will encourage the participants to take the certification exam in the future now that they have some experience with the type of questions that could be asked. 


\section{Summary and Conclusions}

Nurses having the knowledge of sepsis care is very important in the critical care setting because identifying the disease process and initiating treatment in a timely manner can be key to a patient's survival. Having a collaborating approach including all professionals can be proven beneficial to the patient. A need was identified for education related to sepsis in the critical care setting for the nursing staff that work on the critical care unit at Yale New Haven Health-Westerly Hospital. This was identified due to the hiring of several novice nurses that did not have any critical care experience prior to being hired. An educational program on sepsis in the critical care setting was created and a pre- and post-tests were created to assess baseline and post-educational intervention knowledge. As seen in Table 1, the mean scores of the pre- and post-tests improved from $52 \%$ to $78.6 \%$. Displaying a $26.6 \%$ increase in mean score.

Fifteen nurses out of a potential twenty nurses working on the critical care unit completed the pre-test and post-test component. All participants that completed a pre-test also completed a post-test. Testing results were also broken down by years of critical care experience to conclude whether experience influenced testing scores. All fifteen participants were in two different brackets of experience which included " $1-5$ years" and ">10 years." The mean score of the $1-5$ years of experience group which was composed of ten participants was $77.5 \%$. For the $>10$ years of experience group which included five participants was $81 \%$ for the post-test. The participants with the $1-5$ years of experience showed to have a higher increase in mean score from the pre-test to the post-test which was $27 \%$, compared to the participants with $>10$ years of experience which was $26 \%$. 
Limitations to this project were evident. Since the potential sample size was only twenty to start there was a potential to have a small sample size. Fifteen out of the potential twenty nurses $(75 \%)$ decided to participate and completed the pre- and the posttest. The delivery of the education and the testing was not done in a controlled setting but the nurses were assured that testing results were completely confidential and that this project was only to their benefit. The participants expressed multiple times to the author that they enjoyed the fact that they could take the tests and the education home with them to complete on their own time.

In conclusion, this program development project was successful in increasing the knowledge of the critical care nursing staff related to sepsis. The staff found it beneficial and by reviewing the mean scores of the pre- and post-test it is evident that the knowledge of the participants improved. 


\section{Recommendations and Implications for Advanced Nursing Practice}

This program development project highlighted the need for education on treating the patient with sepsis, specifically with septic shock in the critical care setting. The results of the project between the pre- and post-test demonstrated an increased knowledge by the participants. The advanced practice registered nurse (APRN) is in a unique position to enable educational programs and assess educational needs of a certain department. Utilizing the role of the APRN that encompasses a holistic outlook on nursing, the APRN student had the ability to realize that there was a need for sepsis education, especially on a unit that the APRN student has worked with the participants on.

Providing this education on sepsis care could be used in future orientations for new nurses working in their first critical care setting. Education should not only be considered for the novice nurses, but for all nurses throughout the experience spectrum. Nursing care is constantly evolving and it is the nurse's obligation to keep current with the most evidence-based care that they can provide to their patients. The APRN can deliver education to staff nurses throughout the unit but also to contribute to the progress and maintenance related to policy of that unit or system. 


\section{References}

Agrawal, A., Gupta, A., Consul, S., \& Shastri, P. (2011). Comparative study of dopamine and norepinephrine in the management of septic shock. Saudi Journal of Anaesthesia, 5(2), 162-166. http://doi.org/10.4103/1658-354X.82784

Aminzadeh, Z., \& Parsa, E. (2011). Relationship between age and peripheral white blood cell count in patients with sepsis. International Journal of Preventive Medicine, 2(4), 238-242.

Borer, A., Brotfain, E., Fucs, L., Galante, O., Koyfman, L., Klein, M., \& Toledano, R., (2016). Positive fluid balance as a major predictor of clinical outcome of patients with sepsis/septic shock after ICU discharge. American Journal of Emergency Medicine, 34(11), 2122-2126. doi:10.1016/j.ajem.2016.07.058

Bouza, E., Bunsow, E, Burillo, A., Muñoz, P., Sanchez, C., \&., Vecchio, (2015). Improved sepsis alert with a telephone call from the clinical microbiology laboratory: A clinical trial. Medicine, 94(39), e1454. http://doi.org/10.1097/MD.0000000000001454.

Carneiro, A., Pereira, A., Póvoa, P., \& Ribeiro, O., \& (2009). Influence of vasopressor agent in septic shock mortality. Results from the Portuguese community-acquired sepsis study (SACiUCI study). Critical Care Medicine, 37(2), 410-416. doi:10.1097/CCM.0b013e3181958b1c.

De Backer, D., Biston, P., Devriendt, J., Madl, C., Chochrad, D., Aldecoa, C., \& ... Vincent, J. (2010). Comparison of dopamine and norepinephrine in the treatment of shock. New England Journal of Medicine, 362(9), 779-789 11p. doi:10.1056/NEJMoa0907118. 
Delaney, M. M., Friedman, M. I., Dolansky, M. A., \& Fitzpatrick, J. J. (2015). Impact of a sepsis educational program on nurse competence. Journal of Continuing Education In Nursing, 46(4), 179-186. doi:10.3928/00220124-20150320-03

Dennison, R. (2013). Pass CCRN! (4th ed.). Pages 685-740, St. Louis, MO: Elsevier. Dunkley, S., \& McLeod, A. (2015). Neutropenic sepsis: Assessment, pathophysiology and nursing care. British Journal of Neuroscience Nursing, 11(2), 79-87.

El Solh A, Akinnusi M, Alsawalha L, \& Pineda L, 2008. Outcome of septic shock in older adults after implementation of the sepsis 'bundle'. Journal of The American Geriatrics Society [serial online]. February 2008;56(2):272-278. Available from: CINAHL Plus with Full Text, Ipswich, MA. Accessed August 6, 2017.

Hansen, G. (2013). Blood cultures and the detection of sepsis. MLO: Medical Laboratory Observer, 45(6), 42-43

Hariyanto, H., Yahya, C. Q., Widiastuti, M., Wibowo, P., \& Tampubolon, O. E. (2017). Fluids and sepsis: Changing the paradigm of fluid therapy: A case report. Journal of Medical Case Reports, 111-7. doi:10.1186/s13256-016-1191-1.

Iwashyna, T. J., Ely, E. W., Smith, D. M., \& Langa, K. M. (2010). Long-term cognitive impairment and functional disability among survivors of severe sepsis. Jama, 304(16), 1787-1794. http://doi.org/10.1001/jama.2010.1553

Jensen, J. U., Heslet, L., Jensen, T. H., Espersen, K., Steffensen, P., \& Tvede, M. (2006). Procalcitonin increase in early identification of critically ill patients at high risk of mortality*. Critical Care Medicine,34(10), 2596-2602.

doi:10.1097/01.ccm.0000239116.01855.61. 
Kleinpell, R. (2017). Promoting early identification of sepsis in hospitalized patients with nurse-led protocols. Critical Care, 211-3. doi:10.1186/s13054-016-1590-0.

Martin, G., Mannino, D., Moss, M., Martin, G. S., Mannino, D. M., \& Moss, M. (2006). The effect of age on the development and outcome of adult sepsis. Critical Care Medicine, 34(1), 15-21.

Marty, P., Roquilly, A., Vallée, F., Luzi, A., Ferré, F., Fourcade, O., ... Minville, V. (2013). Lactate clearance for death prediction in severe sepsis or septic shock patients during the first 24 hours in Intensive Care Unit: An observational study. Annals of Intensive Care, 3, 3. http://doi.org/10.1186/2110-5820-3-3

McDonald KM, Sundaram V, Bravata DM, Lewis R, Lin N, Kraft SA, McKinnon M, Paguntalan H, Owens DK, (2007). Closing the quality gap: A critical analysis of quality improvement strategies. Care Coordination, 7, pages 1-6.

Niazi, J. Nash, T Seoane, L., Sundell, E. \& Winterbottom, F. (2011). Improving sepsis outcomes for acutely ill adults using interdisciplinary order sets. Clinical Nurse Specialist: The Journal for Advanced Nursing Practice, 25(4), 180-185. doi:10.1097/NUR.0b013e318221f2aa

Peel, M. (2008). Care bundles: Resuscitation of patients with severe sepsis. Nursing Standard, 23(11), 41-46.

Perez, C. (2015). Effective monitoring of patients with sepsis. RT: The Journal for Respiratory Care Practitioners, 28(9), 20-23

Picard, K., O'Donoghue, S., Young-Kershaw, D., \& Russell, K. (2006). Development and implementation of a multidisciplinary sepsis protocol. Critical Care Nurse, 26(3), 43-54. 
Rhodes, A., Evans, L., Alhazzani, W., Levy, M., Antonelli, M., Ferrer, R., \& ... Backer, D. (2017). Surviving Sepsis Campaign: International guidelines for management of sepsis and septic shock: 2017. Intensive Care Medicine, 43(3), 304-377. doi:10.1007/s00134-017-4683-6

Robson, W. (2008). The Sepsis Six: Helping patients to survive sepsis. British Journal Of Nursing, 17(1), 16-21.

Sakr, Y., Birri, P. R., Kotfis, K., Nanchal, R., Shah, B., Kluge, S., \& ... Rubatto Birri, P. N. (2017). Higher fluid balance increases the risk of death from sepsis: Results from a large international audit. Critical Care Medicine, 45(3), 386-394. doi:10.1097/CCM.0000000000002189

Sepsis Alliance. (2017). Sepsis information guide. Retrieved July 12, 2017, from http://www.sepsis.org/resources/sepsis-information-guides/\#gf_9

Talebi-Taher, M., Babazadeh, S., Barati, M., \& Latifnia, M. (2014). Serum inflammatory markers in the elderly: Are they useful in differentiating sepsis from SIRS?. Acta Medica Iranica, 52(6), 438-442. Retrieved from http://acta.tums.ac.ir/index.php/acta/article/view/4783

Vaughan, J., \& Parry, A. (2016). Assessment and management of the septic patient: Part 2. British Journal of Nursing, 25(21), 1196-1200

Yousefi, H., Nahidian, M., \& Sabouhi, F. (2012). Reviewing the effects of an educational program about sepsis care on knowledge, attitude, and practice of nurses in intensive care units. Iranian Journal of Nursing and Midwifery Research, 17(2 Suppl1), S91-S95. 


\section{Appendix A \\ Chief Administrator Officer Approval Letter}

To Rhode Island College of Nursing (MSN) Department

Matthew Willard has permission to educate and test registered nurses at Lawrence and Memorial Westerly Hospital regarding sepsis care in the critical care setting. I understand this will take place during the fall/early winter months of 2017-2018.

Thank you,

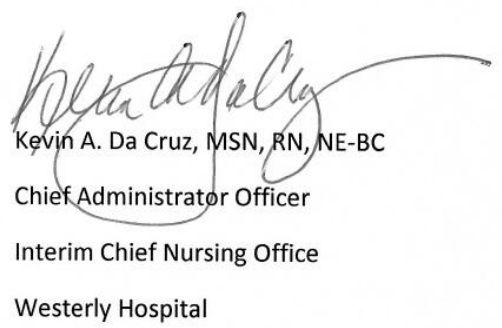




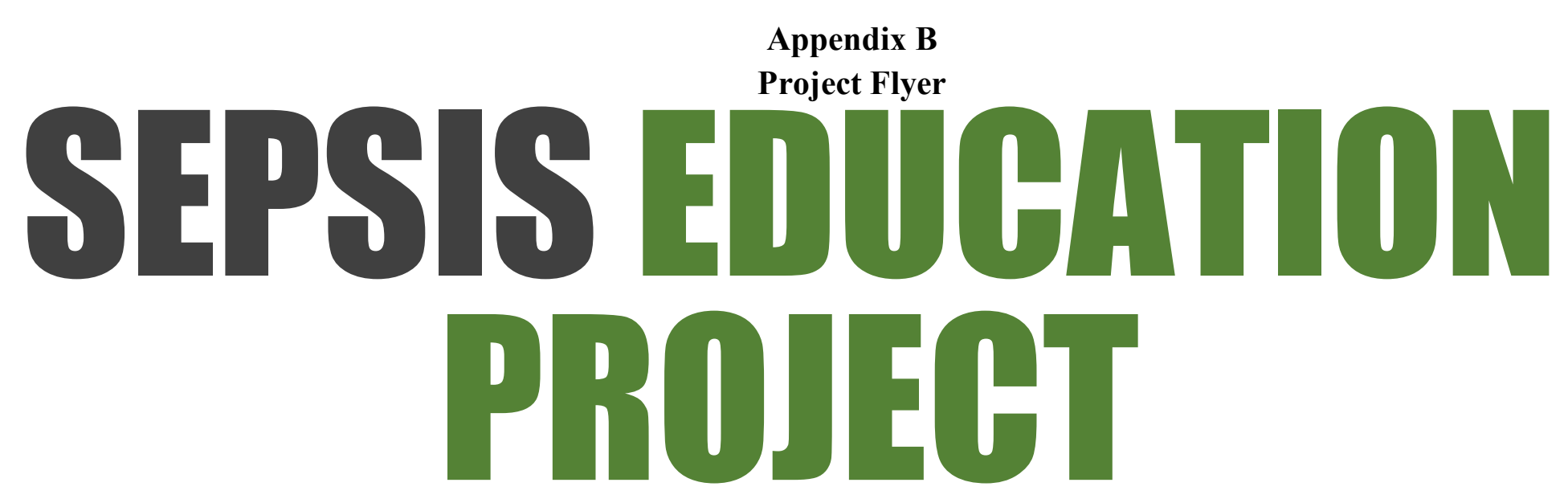

When: Starting November through December, 2017

How does it work?

1. One week to complete a 20 multiple-choice pre-test questionnaire on sepsis in the critical care setting.

2. One week of sepsis education. All educational material will be presented in a personal hinder for the staff to take a copy and review on their own. The material will be found in the break room.

3. One week to complete a post-test questionnaire

GOAL/PURPOSE OF THIS PROJECT:

IMPROUE THE KNOWLEDGE OF SEPSIS IDENTIFICATION AND TREATMENT BY PROUIDING EDUCATION TO THE GRITIGAL GARE NURSING STAFF

\section{PARTIPEATION IS GOMPLETELY VOLUNTARY!}

FOR QUESTIONS:

CONTAGT: MATTHEW WILLARD

GELL: 401-256-4665

EMAIL:MWILLARD_0087@EMAILRIC.EDU 


\section{Appendix C Staff Email}

To all critical care nursing staff at Yale Westerly Hospital,

I am Matthew Willard, an Acute Care Nurse Practitioner student at Rhode Island College. I would like to inform you of an educational opportunity on the topic of sepsis care in the critical care setting. All registered nursing staff at the Yale Westerly Hospital that work in the critical care setting are identified as possible participants for this educational opportunity. The purpose of this program development project is to improve the knowledge of sepsis identification and treatment by providing education to the critical care nursing staff. The project will begin midNovember and run through December, 2017. If you choose to participate in this project, you will be asked to complete three components. The components will include pre-testing, an educational program, and post-testing. Since nursing is such a busy profession, the opportunity to complete these three components on your own schedule will be available. Participation is voluntary and will not affect your position.

The first week of the project will include pre-testing. Pre-testing will last one week and during that week, participants will complete a 20 -question multiple choice questionnaire which will be located in the break room in a designated envelope. The test will be returned in the box marked "Completed Pre-tests," which will also be located in the break-room. Week 2 will include the education component. A binder will be created for each of you that will include information on certain topics of sepsis as well as information that was tested on in the pre-test. The binder can be reviewed on your own whenever you may have time that week. Bulletin boards in the breakroom will also contain information for review. Week 3 will include post-testing. The post-test procedure will follow the same as the pre-test. Completed tests will be returned to the designated box labeled "Completed Post Tests."

Participation in this project will assume consent. All testing will be anonymous. Participants will use a unique identifier on the last page of the pre- and post-tests so results can be compared from pre- and post-testing. The participant's unique identifier will be their mother's birthday, including the month and day (ex. 10/24) in the designated area on the last page in the lower left hand corner. Please do not write your name anywhere on either test. Next to where you will write your mother's birthday will be a section where you can write how many years of critical care experience you have. This will be used for trending results. The goal of the project is to provide information to the staff on sepsis

If you have any questions or concerns about the research or educational component, you can contact Matthew Willard at mwillard 0087@email.ric.edu or by cell phone at 401-256-4665 or Debra Servello at dservello@ric.edu. If you have any questions about your rights as a research subject, please feel free to contact the IRB designate at Rhode Island College at IRB@RIC.edu.

Thank you for your time,

Matthew Willard, RN-BSN, CCRN 


\section{Appendix D \\ Pre/Post Test Questions}

1. A 70-year-old man presents to the emergency department with a 2-day history of fever, chills, cough, and right-sided pleuritic chest pain. On the day of admission, the patient's family noted that he was more lethargic and dizzy and was falling frequently. The patient's vital signs are: temperature, $101.5^{\circ} \mathrm{F}$; heart rate, $120 \mathrm{bpm}$; respiratory rate, 30 breaths/min; blood pressure, 70/35 mm Hg; and oxygen saturation as measured by pulse oximetry, $80 \%$ without oxygen supplementation. A chest radiograph shows a right lower lobe infiltrate. What is the first step in the initial management of this patient?

A. Antibiotic therapy

B. $\beta$-Blocker therapy to control heart rate

C. Intravenous (IV) fluid resuscitation

D. Supplemental oxygen and airway management

E. Vasopressor therapy with dopamine

2. Which of the following is an indication for using corticosteroids in septic shock?

A. Acute respiratory distress syndrome (ARDS)

B. Necrotizing pneumonia

C. Peritonitis

D. Sepsis responding well to fluid resuscitation

E. Vasopressor-dependent septic shock

\section{The following description pertains to questions $3,4, \& 5$ :}

An elderly patient is admitted with pneumonia. This morning he is febrile, tachycardic, tachypneic, and confused.

3. Which of the following would be indications of sepsis?

A. Hypotension and tachycardia

B. Tachypnea and leukocytosis

C. Increased systemic vascular resistance and vasoconstriction

D. Hypothermia and leukopenia

4. Which of the following is the initial response of the cardiovascular system to sepsis?
A. Bradycardia
B. Decreased contractility
C. Increased cardiac output
D. Increased preload 
5. The elderly patient is now started on norepinephrine (Levophed). You would monitor him closely for which of the following?
A. Hypotension
B. Abdominal Pain
C. Bronchospasm
D. Bradycardia

6. Which of the following is not a common pathophysiologic manifestation seen in patients with septic shock?

A. Myocardial depression

B. Maldistribution of blood flow

C. Tissue hypoxia resulting from decreased oxygen delivery

D. Coagulopathy

7. Which of the following statements about hypotonic solutions is accurate?

A. They move fluid from the interstitial space to the intravascular space.

B. They are given to patients with third-space fluid shift.

C. They increase perfusion to vital organs.

D. They increase interstitial and intracellular volume.

8. Which of the following is characteristic of the early stage of septic shock?

A. Increased cardiac output/cardiac index (CO/CI), decreased systemic vascular resistance (SVR), increased venous oxygen saturation (SvO2)

B. Decreased CO/CI, increased SVR, decreased SvO2

C. Increased $\mathrm{CO} / \mathrm{CI}$, increased SVR, increased SvO2

D. Decreased CO/CI, decreased SVR, decreased SvO2

\section{The following situation pertains to questions to questions $9 \& 10$ :}

A 65-year-old man is admitted to the critical care unit with a diagnosis of septic shock.

He has been receiving chemotherapy for lung cancer. His skin is warm and dry, and he is restless. His white blood cell count is elevated above normal. Hemoglobin, hematocrit, and red blood cell count are normal. Vital signs are blood pressure $80 / 50 \mathrm{~mm} \mathrm{Hg}$, heart rate 120 beats/min and regular, respiratory rate 32 breaths $/ \mathrm{min}$ and regular, and temperature $39^{\circ} \mathrm{C}$.

Arterial blood gases reveal the following:

$\mathrm{pH}$

$\mathrm{PaO} 2$

$\mathrm{PaCO} 2$

HCO3

Oxygen saturation
7.25

$60 \mathrm{~mm} \mathrm{Hg}$

$25 \mathrm{~mm} \mathrm{Hg}$ $13 \mathrm{mEq} / \mathrm{L}$ 
9. What hemodynamic alteration should the nurse anticipate?
A. Decreased cardiac output (CO)
B. Increased venous oxygen saturation (SvO2)
C. Increased systemic vascular resistance (SVR)
D. Increased oxygen consumption

10. Dobutamine is started at $10 \mathrm{mcg} / \mathrm{kg} / \mathrm{min}$. Normal saline is infusing at $150 \mathrm{~mL} / \mathrm{hr}$. Which of the following would be most indicative of improvement in this patient?
A. Increase in venous oxygen saturation (SvO2)
B. Decrease in arterial lactate
C. Increase in cardiac output
D. Increase in urine output

11. A 44-year-old woman, diagnosed with sepsis, develops petechiae, ecchymosis, mucosal oozing, and hematuria. Her prothrombin time (PT) and activated partial thromboplastin time (aPTT) are prolonged, but she is not receiving anticoagulant therapy. The most likely cause of these changes is:

A. Hepatic failure.

B. Systemic inflammatory response syndrome (SIRS).

C. Disseminated intravascular coagulation (DIC).

D. Platelet dysfunction.

12. Your patient is in septic shock. Which of the following would alert you that he is at risk of acute tubular necrosis?
A. Serum potassium of $5 \mathrm{mEq} / \mathrm{L}$
B. $\mathrm{pH}$ of 7.36
C. Urine output of $50 \mathrm{~mL} / \mathrm{hr}$
D. Mean arterial pressure (MAP) of $58 \mathrm{~mm} \mathrm{Hg}$

13. Which of the following organ systems is among the most common to fail in severe sepsis?
A. Renal
B. Hematologic
C. Hepatic
D. Cardiovascular 
14. You are taking care of an 80yo male originally admitted in septic shock secondary to a RLL PNA on a mechanical ventilator in ARDS. Which are the most beneficial ventilator settings for a patient in ARDS?

A. HIGH tidal volume, HIGH PEEP

B. LOW tidal volume, LOW PEEP

C. LOW tidal volume, HIGH PEEP

D. HIGH tidal volume, LOW PEEP

15. Which of the following is the most important aspect of patient management related to Septic shock?

A. Early initiation of broad-spectrum antibiotics

B. Limitation of length of stay in a critical care unit

C. Avoidance of invasive catheters and procedures

D. Recognition of high-risk patients and early manifestations of organ dysfunction.

16. Which of the following is not an integral aspect of the pathophysiology of severe sepsis?

A. Immunosuppression

B. Excessive coagulation

C. Impaired fibrinolysis

D. Exaggerated inflammation

17. A nursing home patient presents to emergency room with fever, cloudy urine, and chest pain. Vital signs: 104.7degrees F, HR Afib 150's, BP 70/40's. Which of the following is inappropriate?

A. Give fluids

B. Draw cultures and start antibiotics

C. Place a central line

D. Give nitroglycerin for chest pain

18. Risk factors that are typically linked to sepsis include

A. Patients with chronic health problems.

B. Patients taking immunosuppressant drugs.

C. The increasing number of elderly patients.

D. All the above 
19. Which common laboratory test shows early warning of sepsis, even before clinical suspicion?
A. Urinalysis
B. PT/PTT
C. ESR
D. CBC

20. Regarding hemodynamic support, which of the following agents is the best initial therapeutic intervention?
A. 5\% albumin
B. Lactated ringer solution
C. Normal saline
D. Norepinephrine

(Pass CCRN!, Dennison, 685-740)

\section{Mother's Birthday (Month/Day):}

Years of Critical Care Experience: Please Circle One of The Following

$<1$ year $\quad 1-5$ years $\quad 6-10$ years $\quad>10 y e a r s$ 


\section{Appendix E}

Objectives

Upon competition of the educational program the participants will:

1. Increase confidence in identifying sepsis and the progression to septic shock in the critical care setting.

2. Demonstrate knowledge about hemodynamic monitoring and interventions needed in the hemodynamically unstable patient with septic shock evidenced by completing education requirements and improved post-testing results compared to pre-testing results.

3. Describe the treatment of sepsis in the elderly population.

4. Describe the importance of the sepsis laboratory testing required to help tailor sepsis treatment.

5. Identify sepsis protocol interventions that are crucial in treatment. 


\begin{abstract}
Appendix F
Answers and Rational

1. Answer: D. Rational: The initial evaluation of any critically ill patient in shock should include assessing and establishing an airway, evaluating breathing (which includes consideration of mechanical ventilator support), and restoring adequate circulation. Adequate oxygenation should be ensured with a goal of achieving an arterial oxygen saturation of $90 \%$ or greater.
\end{abstract}

2. Answer: E. Rational: An inappropriate cortisol response is not uncommon in patients with septic shock. Low-dose IV corticosteroids (hydrocortisone 200-300 mg/day) are recommended in patients with vasopressor-dependent septic shock.

3. Answer: D. Rational: Sepsis is infection with systemic inflammatory response syndrome (SIRS). Hypothermia and consumptive leukopenia are evidence of SIRS.

4. Answer C. Rational: Contractility and cardiac output are increased in sepsis and early septic shock. The heart rate increases. Preload and afterload decrease because of massive vasodilation.

5. Answer: B. Rational: Norepinephrine stimulates predominantly alpha receptors but also stimulates beta receptors. Monitor the patient closely for indications of excessive vasoconstriction and ischemia, such as acrocyanosis, chest pain, abdominal pain, and decreased urine output.

6. Answer: C. Rational: In septic shock the delivery of oxygen to the tissues is increased, but extraction and use of oxygen are impaired.

7. Answer: D. Rational: Hypotonic solutions are hypotonic to blood, so they move from the intravascular space to the interstitial space and intracellular space. 
8. Answer: A. Rational: The early stage of septic shock sometimes is called the hyperdynamic or warm phase. The cardiac output and index are increased, the SVR is decreased, and the $\mathrm{SvO}_{2}$ is increased. Consider that vasodilatory mediators dominate the early stage of septic shock, and vasoconstrictive catecholamines dominate the late stage. The $\mathrm{SvO}_{2}$ is increased in early septic shock because the tissues are unable to extract the oxygen from the blood. Therefore, the venous blood is more saturated than normal when it returns to the lungs for reoxygenation.

9. Answer: B. Rational: This case study describes hyperdynamic septic shock. In this early stage of septic shock, the CO is increased and SVR is decreased. Oxygen delivery is normal or even increased in this stage, but tissue extraction is decreased. Because the tissues cannot extract the oxygen from the blood, the oxygen saturation of the venous blood $\left(\mathrm{SvO}_{2}\right)$ is increased.

10. Answer: B. Rational: Oxygen delivery is being increased by dobutamine and normal saline infusions, but the real issue is whether the tissues have been able to extract the oxygen being delivered. An increase in $\mathrm{SvO}_{2}$ would indicate that even less oxygen is being extracted from the arterial blood. An increase in cardiac output and urine output indicate that oxygen delivery is increased but do not indicate whether the tissues have been able to extract the oxygen. Lactic acid is produced by anaerobic metabolism, and arterial lactate will be decreased if the tissues convert back to aerobic metabolism 11. Answer: C. Rational: Petechiae indicate that there is a decrease in the number or effectiveness of the platelets. However, a decrease in platelets does not affect PT and aPTT, which are increased. In addition, there is bleeding in a patient who has not previously had a coagulopathy and is not receiving anticoagulants. This is DIC. 
12. Answer: D. Rational: MAP of less than $60 \mathrm{~mm} \mathrm{Hg}$ for as short a period as 40 minutes may cause acute tubular necrosis and acute kidney injury.

13. Answer: B. Rational: Coagulopathies are seen early and universally in severe sepsis. 14. Answer: A: Rational: A mean arterial pressure of less than $60 \mathrm{~mm} \mathrm{Hg}$ for as short a period as 40 minutes may result in acute tubular necrosis and acute kidney injury.

Prolonged hypoperfusion is the most likely cause of acute tubular necrosis in a patient with MODS.

15. Answer: D. Rational: Recognition of high-risk patients and monitoring for early manifestations of organ dysfunction is the most important aspect of management related to MODS.

16. Answer: A. Rational: Although immunosuppression is a major risk factor for sepsis, it is not an integral aspect of the pathophysiology.

17. Answer: A. Rational: DIC is initiated by massive tissue and/or endothelial injury. Microclots develop that may cause ischemia distal to the clots and may deplete clotting factors. These clots trigger the fibrinolytic system, causing development of fibrin split products, which have their own anticoagulant effect. Thus stable clots are broken down, and there is an inability to form stable clots.

18. Answer: D. Rational: all these are risk factors

19. Answer: D. Rational: CBC's can offer clinicians critical insight into morphological features of red cells, so that they can further guide their diagnostic work-up, especially in anemic patients.

20. Answer: C. Rational: Normal saline works as a volume expander and used in shock to help the patient become hemodynamically stable. 